\title{
A working memory intervention weakens the reconsolidation of a threat memory and its biases processing towards threat.
}

Soledad Picco ${ }^{1,2}$, Luz Bavassi ${ }^{1,2,3}$, Rodrigo S. Fernández ${ }^{1,2, *}$, María E. Pedreira ${ }^{1,2,{ }^{*}, * *}$

1- Instituto de Fisiología, Biología Molecular y Neurociencias (IFIByNE)-CONICET.

2- Facultad de Ciencias Exactas y Naturales, Universidad de Buenos Aires, Argentina.

3- Departamento de Física, Universidad de Buenos Aires, Argentina.

* These authors contributed equally to this work.

**: Correspondence concerning this article should be addressed to Maria E. Pedreira, IFIByNE (CONICET), Ciudad Universitaria, (1428), Buenos Aires, Argentina. Phone: +5411 4576-3368 (251). Email: mpedreira@fbmc.fcen.uba.ar

\section{Short title:}

\section{Threat-memory weakening and its biases towards threat}

\section{Keywords:}

Reconsolidation; Threat conditioning; Cognitive effort; Emotional /cognitive biases; Memory interference; Experimental psychopathology. 


\section{ABSTRACT}

BACKGROUND: Threat-conditioning (TC) memory plays a central role in anxiety disorders, but not in a simple way. This memory impacts on complex cognitive systems by modifying behavioral responses with a bias to fearful stimuli and overestimating potential threats. In this study we proposed a global approach analyzing the scope of disrupting TC memory reconsolidation in the implicit memory, the declarative contingency and the cognitive biases.

METHODS: Day 1: Subjects were trained on TC. Day 2: after Threat-memory reactivation, one group performed a high demanding working memory task (HWM) and the other a low demanding working memory task (LWM). The last group, only performed the HWM task. Day 3: TC memory was tested by an extinction session followed by reinstatement. Finally, all subjects completed tasks targeting stimuli representation, valuation and attentional bias towards threat.

RESULTS: Disrupting reconsolidation of TC memory with a HWM weakened the implicit memory retention and faded the representation and valuation towards threat but it had no effect on attentional bias. Moreover, we revealed that subjects' performance during the working memory task was specifically associated in TC memory retention.

CONCLUSIONS: Our findings reveal a strong impairment of the threat-memory restabilization and associated emotional biases. This may result from the competition between defensive survival and central-executive control networks. Our results fits with Experimental Psychopathology approach, disentangling the relation between the implicit memory, cognitive, valenced systems and the possibility to weaken both the 
bioRxiv preprint doi: https://doi.org/10.1101/2020.01.08.898643; this version posted January 9, 2020. The copyright holder for this preprint (which was not certified by peer review) is the author/funder. All rights reserved. No reuse allowed without permission.

threatening memory and the systems associated with the maintenance of anxiety profile. 


\section{INTRODUCTION}

Anxiety disorders are the most frequent type of mental disorders being a relevant public health problem, which implies disability in the daily life and causes individual and societal cost $(1,2)$. In this context, experimental models are necessary to understand deeply the nature of them. Considering that fear and anxiety are in the core of these disorders, it is possible to model such symptoms in animals and humans using conserved defense-survival responses (3). In the laboratory, the most useful paradigm is Threat-Conditioning (TC) (4). Thus, specific fear memories can be formed using the repeated pairing of an initially neutral cue (conditioned stimulus, CS) with a naturally aversive stimulus (unconditioned stimulus, US). As a result, an associative threat-memory is acquired. The later presentation of the CS will retrieve the US representation and elicit a conditioned threat response which predicts the occurrence of the US. Threat memory, plays a central role in anxiety disorders but not in a simple way $(5,6)$. The CS predictive value for the negative outcome of the US, involves other components such as exacerbated negative representations associated with anticipated catastrophic consequences (7). The major concern, assuming this oversimplification, relies on the fact that, these defense-survival mechanisms are only one piece of the complex puzzle that contributes to human anxiety and neglects the complex cognitivebehavioral anxiety responses such as biased processing (8-10) towards fearful stimuli and overestimation potential threats $(7,11,12)$.

In the last years, the immutable nature of consolidated memories has been challenged (13-15). After being consolidated, the presentation of a reminder (memory reactivation) of an acquired representation, opens a new round of re-stabilization of 
memory called reconsolidation $(16,17)$. The reconsolidation process enables the update of consolidated memories. After its re-emergence in the field, a frequent proposal appears: the use of the reconsolidation process to modify maladaptive memories (18-21). Thus, different reports reveal that pharmacological interference of memory re-stabilization "erase" the implicit/emotional component of memory leaving the declarative component intact $(22,23)$. This approach also showed to be efficient with a subclinical phobic sample and patients with post-traumatic stress disorders $(24,25)$. A complementary approach, is the use of behavioral interference of memory reconsolidation. For example, the reactivation-extinction procedure uses extinction learning after memory reactivation (26). It was observed that, the negative value of the CS could be weakened (updated) with repeated presentations of the CS alone during the time window of the reconsolidation process. Thus, memory updating by the reconsolidation process reveals its effect on the implicit memory and maintains the declarative component unaffected.

A novel approach in the field suggests that memory reconsolidation depends on active working-memory processing of the reactivated memory (27). The circuits controlling working memory are limited and compete for resource allocation (28). Thus, if a working-memory intervention engages similar resources as those required for memory re-stabilization, the target memory is weakened as a result of the competition. For example, James and coworkers used the visuo-spatial game Tetris as an interference task during the reactivation of an intrusive film memory (27). The competition for visuo-spatial resources impaired the reconsolidation of visual features of intrusive memory. However, using a different task, where the target memory and the 
interference task, depended on the amygdala-based processing, Chalkia and coworkers (29) obtained negative results. That is, the execution of an emotional working memory task after threat-memory reactivation did not impaired memory reconsolidation. A possible explanation for these disparate findings can emerge from fMRI studies. With this technique, it was possible to describe large-scale functional networks, defined by a set of cortical and sub-cortical regions that co-activate together. For example, the salience network responds to threat and also to salient stimuli $(30,31)$. Another described network is central-executive control network. Activation of this network, is observed during cognitively demanding tasks such as working memory maintenance (32). In a recent report it was shown that during acute threat the salience/defensive survival network and the central executive control network act as opposing systems (33).

Anxiety disorders engage physiological, emotional and cognitive changes (34). Consequently, focusing only on the analysis of acquired associative responses at physiological level (i.e. electrodermal activity or startle responses) disregards central features of this multifaceted phenomena, such as the cognitive and negative-valenced domains (35). To overcome these difficulties, we had developed a paradigm of TC, analyzing how this implicit memory could affect different valenced and cognitive systems in healthy participants (36). Subjects first underwent TC and then performed several tasks targeting biased cognitive processing towards threat (i.e. attentional bias) and the valuation systems (i.e. overestimation of threat and their consequences), which commonly underlie sub-optimal decision making in anxiety disorders $(37,38)$. We found that participants rated as more aversive the reinforced stimuli $(\mathrm{CS}+)$ and 
generalized the fear to non-trained stimuli. Additionally, participants overestimated the probability of negative events associated with the CS+ and the reaction-times to CS+ showed an attentional bias towards threat. Overall, we found that TC in a healthy population could generate a similar biased processing towards threats such as those encountered in anxiety disorders.

Thus, we moved a step forward and analyzed the effect of reconsolidation interference of a threat-memory on the negative-valence and cognitive system. Here, we manipulated the demanding aspects of a working memory intervention, using a highly demanding working memory task (HWM, namely the Paced Auditory Serial Addition Task -PASAT: (39)) or a low demanding working memory task (LWM) as interference. We explored the interference effects not only on the implicit memory, but also on the negative valenced and cognitive systems. In this sense, we performed a TC protocol which included three groups. All subjects were trained on TC on Day 1, using male faces as CS's. On Day 2, two groups were exposed to a cue-reminder and performed a HWM task or a low demanding working memory task (LWM, control intervention). The last group, only performed the HWM task. The TC memory was tested on Day 3 by the repeated presentation of the different CSs (extinction session) followed by the presentation of three unsignaled US's (reinstatement). Finally, all subjects completed a set of different tasks targeting the negative-valenced and cognitive systems: stimuli representation (aversiveness) and threat-generalization, valuation (probability and cost) and attentional bias towards threat.

Our working hypothesis had two driven proposals. The first was that considering the competence between the salience and central executive networks for limited 
resources during memory reactivation, we expected that, the impairment of the TC memory re-stabilization would be associated with the demanding levels (high vs low) of the working memory intervention. The second, given the narrow relation between TC memory and the negative-valence and cognitive systems, its interaction might determine how reconsolidation interference may reduce acquired emotional and cognitive biases towards threat.

\section{METHODS AND MATERIALS.}

\section{Participants}

A total of 67 undergraduate and graduate youths (37 females and 30 males) from the University of Buenos Aires (Argentina) mean age of 21,63 $\pm 2,69$ were included in the study. Participants were randomly assigned to one of three groups. All participants gave written informed consent before the experiment, approved by Ethics Committee of the Review Board of the Sociedad Argentina de Investigación Clínica.

\section{Procedure}

After arriving, subjects completed the subjective assessment questionnaires and were randomly assigned to a Reactivation-HWM ( $n=23)$, noReactivation-HWM $(n=23)$ and Reactivation-LWM $(n=21)$ group. On Day 1, participants underwent a TC. Day 2 consisted on a reactivation session of the TC followed by a working memory intervention (interference) or no-reactivation. On Day 3 an extinction was performed as a test of the retention of TC memory, followed by reinstatement. Finally, subjects performed different tasks to assess cognitive and negative-valenced domains. All tasks 
were programmed with Matlab (Mathworks Inc. Sherborn, MA, USA), Psychlab and the Psychtoolbox module.

\section{Subjective assessment}

The State-Trait Anxiety Inventory (STAI-S and STAI-T, (35)) and the Beck Anxiety Inventory (BAI, (36)) questionnaires were used to asses participant's trait-anxiety and the presence of physiological symptoms.

\section{Threat Conditioning}

Acquisition. On Day 1, all participants underwent TC. Each face-stimulus (see supplement) appeared 8 times (24 trials total). One of the face-images (CS+) was followed by a tone-US (75\% of reinforcement), while the other images (CS- and CSn) were not. All the CS's were presented for $6 \mathrm{~s}$ and the US appeared $1,5 \mathrm{~s}$ before CS offset. The inter-trial interval varied between 8-12 s.

Reactivation. On Day 2, two groups (Reactivation-HMW and Reactivation-LMW) received a reactivation session, which consisted of an unreinforced presentation of the CS+, followed by an interruption and a black screen indicating the end of the experiment. After memory-reactivation, groups performed a working memory task (HMW or LMW). The noReactivation-HMW only completed the HMW without memory reactivation.

Extinction and Reinstatement. On day 3, all groups performed a retention test. Extinction consisted in 12 unreinforced presentations of each CS. Then, during 
reinstatement, participants were exposed to 3 consecutive unsignaled US, followed by the presentation of each CSs.

Skin Conductance Response (SCR) was used as measure of the implicit memory (TC) throughout the experiment (see supplement).

\section{US expectancy.}

Declarative memory of TC was assessed using an external keyboard (Yes/No buttons) in which subjects were required to answer the US expectancy during each CS presentation. On each trial, subjects were instructed to press "YES" button if they expected the CS to be followed by the US (tone) or "NO" button if they expected otherwise.

\section{Working Memory Intervention.}

HMW. The Paced Auditory Serial Addition Test (PASAT) (34) was used as the cognitive demanding task (HWM) for the Reactivation-HWM and noReactivation-HMW groups. In the HMW a pre-recorded series of numbers (1 to 9$)$ were presented through headphones and subjects were asked to add the last two numbers. The task consisted in 61 trials (additions) and numbers were presented with a $2 \mathrm{~s}$ interval. Only answers given within the interval were considered as correct For example, if the digits presented were ' 2 ', ' 4 ' and ' 8 ', the correct answer would be first ' 6 ' and then ' 12 '. 
LMW. For Reactivation-LMW group, the task consisted in the same pre-recorded audio as the HMW, but subjects were asked to answer within a $2 \mathrm{~s}$ interval if the digits were even or odd.

The demanding aspects of the working memory interventions were analyzed trough arousal and performance measures. Thus, arousal was assessed using SCL and it is reported as the mean $(\mu \mathrm{S})$ tonic signal during the tasks. In addition, the proportion of correct responses was used as a measure of working memory performance.

\section{Cognitive and negative-valenced systems.}

Stimuli Representation and Generalization. Before TC on Day 1, and after its evaluation on Day 3, subjects rated the aversiveness of 12 different pictures of males faces using a 0-8 scale (5 angry, 4 neutrals and the ones used as CS+, CS- and CSn; (31)). Pictures were randomly presented on the screen until a response was given with the keyboard. For each group, the mean difference score between post-pre TC, was considered as the Stimuli representation score for CS+ and CS- and the mean difference (post-pre) of neutral and negative faces was considered as the Stimuli Generalization score.

Valuation (Probability and Cost). On day 3, subjects read in the screen 24 positive and 24 negative scenarios, involving either the CS+ or the CS- along its picture $(3,4)$. For each scenario and CS, subjects first responded how likely was the hypothetical event (in a 0 to 8 scale) and then how good/bad it would be for them, using the same 0 to 8 scale. For example, a negative event could be: "How likely it would be you having problems at work with HIM?" and then: "How bad it would be for you having problems 
at work with HIM?". A positive situation could be: "How likely it would be HE taking you to the airport?", and then: "How good it would be HE taking to the airport?". A probability score and a cost score were calculated separately as the mean score difference between positive and negative scenarios for CS+ and CS-.

Attentional bias. On Day 3, after the extinction task (Figure 1), subjects performed a pictorial dot-probe task involving the CS's (36). First, a fixation cross was presented in the center of the screen for 500 ms, followed by a simultaneous display of two CS (left / right) for $500 \mathrm{~ms}$. Then CSs pictures vanished and a small white probe appeared in the previous location (left/right) occupied by one of the CS for $1000 \mathrm{~ms}$. Participants were asked to press a button as fast as they could, according to where they have detected the white probe (left/right) using an external keypad. Four types of stimuli compounds were used: 1) CS+ vs CS-; 2) CS+ vs CSn; 3) CS- vs CSn and 4) two neutral new faces (filler trials). We considered Congruent trials when the probe appeared on the CS+ location (stimuli compounds 1 and 2) or CS- (stimulus compound 3) and Incongruent trials the remaining possibilities (probe on CS- or CSn on compounds 1 and 2; and CSn on compound 3). Probe position (left/right) and face location was counterbalanced. Subjects performed a total of 160 trials in two blocks of 80 (40 trials per condition). Mean reaction times (RT) from the dot-probe task was estimated per condition taking the different between incongruent and congruent trials. Errors of commission, omission or responses less than 150 ms or greater than 2000 ms were not included in analysis. 


\section{Statistical Analysis.}

Subjective assessment and the proportion of correct responses during the working memory tasks (HWM and LWM) were analyzed using a One-Way ANOVA. SCR and proportion of YES responses for US expectancy during TC and extinctionreinstatement, were analyzed by means of a mixed ANOVA for repeated-measures with Group as the between-subjects factor and Stimulus (CS+, CS- and CSn) and Trial, as within-subject factors. When the interaction was significant, simple effects were performed. When sphericity was not accomplished, Greenhouse-Geisser correction was applied. Cognitive and negative-valenced systems were analyzed by separate Mixed-ANOVA's (Group x Stimuli as factors), followed by post-hoc pairwise comparison using the Bonferroni correction for the main effects. Significant interactions were analyzed with simple effects and post-hoc Tukey comparisons.

\section{RESULTS.}

\section{Assessment.}

Participants did not differ in self-reported anxiety or working-memory capacity (see supplement, Table S1).

\section{Reconsolidation interference.}

Analysis of variance revealed a successful threat conditioning in all groups during acquisition on Day 1 (Figure 2; Mixed repeated-measures ANOVA, Stimulus: $F_{2.74}=15.994, p<0.001, \eta p^{2}=0.30 ;$ Stimulus $\times$ Trial Interaction: $F_{8.296}=2.580, p<0.05$, $\left.\eta p^{2}=0.06\right)$. There was a differential increase in SCR amplitudes for CS+ relative to CS- 
and CSn, from the first to the last trials of acquisition in all groups (last trial simple effects, $C S+$ vs $\left.\mathrm{CS}_{\text {all }} \mathrm{p}<0.001\right)$. As seen in Figure 2, a HWM after memory reactivation successfully interfered the re-stabilization of threat memory and prevented the return of fear. On Day 3, the group whose CS+ memory was reactivated and then performed the HWM task (Reactivation-HWM group) showed no differential SCR responding to none of the CS's from the first trial of extinction (last trial of acquisition vs first extinction trial $p<0.05$, Group $\times$ Time interaction $F_{2.144}=2.063, p<0.05$, simple effects, $\left.\mathrm{CS}+\mathrm{vs} \mathrm{CS}_{\text {all }} \mathrm{p}>0.05\right)$. Moreover, the $\mathrm{SCR}$ presented no recovery after reinstatement (simple effects, $\mathrm{CS}+\mathrm{vs} \mathrm{CS}_{\text {all }} \mathrm{p}>0.05$ ). In contrast, both the noReactivation-HWM and the Reactivation-LWM groups demonstrated threat-memory retention from Day 1 to Day 3 (simple effects, $C S+p>0.05$ ) and differential responding to the CS+ in the first trial of extinction (simple effects, $\mathrm{CS}+\mathrm{vs} \mathrm{CS}_{\text {all }} \mathrm{p}<0.05$ ). Moreover, the last trial of the extinction session showed no difference in SCR levels between CS+, CS- and CSn ( $p>0.05)$. Finally, both groups showed threat-memory recovery after reinstatement (simple effects, CS+ vs $\left.\mathrm{CS}_{\text {all }} \mathrm{p}<0.05\right)$. Consistent with previous results $(23)$, reconsolidation interference of the implicit threat-memory did not affect the declarative component of this memory (see supplement, Figure S1, US expectancy).

\section{Working-Memory Intervention.}

Electrodermal activity $(\mathrm{SCL})$ and subject's performance, confirmed the cognitivedemanding nature of the HWM. On Day 2, groups that received the HWM (Reactivation-HWM and noReactivation-HWM) exhibited higher arousal (SCL levels) (Figure $3 \mathrm{~B}, \mathrm{~F}_{2}=4.154, \mathrm{p}<0.001, \eta \mathrm{p}^{2}=0.190$ ) and had a worse performance during the task $\left(F_{2}=43.40, p<0.001, n p^{2}=0.583\right)$, relative to the group which performed the LWM 
(Reactivation-LWM). In order to disentangle the effect of the working memory intervention on the reactivated memory, we performed a correlation analysis including Reactivation-HMW and Reactivation-LMW (Figure 3C). As observed in Figure 3D, arousal and performance during working memory interventions were negatively correlated $(r=-0.37, p<0.05)$, as high levels of $S C L$ were associated with lower proportion of correct responses. Notably, subject's performance predicted changes in threat memory retention between Day 3 and Day 1 (mean SCR difference between first extinction trial and last acquisition trial), (Figure $3 E, r=0.41, p<0.001$ ). Finally, we found no connection between arousal and changes in threat memory retention.

\section{Cognitive and negative-valenced systems.}

Stimuli Representation. After threat-memory evaluation on Day 3, the ReactivationHWM group, rated similarly the aversiveness of the CS+ and the CS- (Figure 4, Stimulus $F_{1.64}=12.074, p<0.001, n p^{2}=0.159$, pairwise comparison $\left.p>0.05\right)$. In contrast, the noReactivation-HWM and the Reactivation-LWM groups, represented the CS+ significantly more aversive and unpleasant than the CS- (Figure $4, p<0.05$ for both groups).

Stimuli Generalization. Subjects rated the aversive faces higher than the neutral faces (Stimulus $F_{1.64}=4.009, p<0.05, n p^{2}=0.05$ ), but we did not found difference between stimulus for each group (Figure 4, Stimulus x group $F_{2.64}=0.031, p>0.05, n p^{2}=0.001$ ). 
Valuation. A Mixed-ANOVA revealed that the Reactivation-HWM group, assigned a similar probability (Stimulus $\mathrm{F}_{1.728}=7.156, \mathrm{p}<0.01, \eta \mathrm{p}^{2}=0.01$ ) and cost (Stimulus $F_{1.729}=6.698, p<0.01, n p^{2}=0,009$ ) to $C S+$ and CS- (pairwise comparison $p>0.05$ ) in either positive or negative scenarios (Figure 4). On the contrary, noReactivation-HWM and Reactivation-LWM groups, estimated higher probability to the CS+ relative to the CS( $p<0.05$ for both groups) and its associated cost ( $p<0.05$ for both groups).

Attentional bias. We found no evidence of attentional bias toward threat in the dotprobe task. A Mixed-ANOVA revealed a similar performance in all groups and no difference between them (Figure 4, Stimulus $F_{2.108}=1.433, p>0.05, \eta p^{2}=0.02$; Stimulus $x$ Group $\left.\mathrm{F}_{4.108}=0.89, \mathrm{p}>0.05, n \mathrm{p}^{2}=0.003\right)$. Moreover, $\mathrm{RT}$ did not differ from zero in any condition (see supplement, Table S2).

\section{DISCUSSION}

Threat-conditioning (TC) is generally an adaptive and self-preserving form of learning which generates an implicit memory. However, it may become a source of pathologies when the reactivity to threat-cues persists in the absence of the reinforcement (7). Initially, this type of learning has been associated with development of anxiety disorders $(6,40)$. Different pieces have been added to the puzzle, to understand this type of disorders such as incubation of fear, failure to inhibit the fear response to safety cues and stimulus generalization (37). Considering this background, in the present report, we demonstrated how the impairment of TC memory re-stabilization by a HMW, affected the implicit memory but not the declarative memory (Figure S1). 
In addition, we revealed that not any working memory task interfere memory reconsolidation because the interference effect depends on the level of cognitive demand (Figure 3) (28). Finally, we showed how the interference of memory reconsolidation reduced biased processing towards threat (i.e. stimuli representation and valuation). Figure 4).

Previously, we demonstrated that attentional bias towards threat could be acquired and maintained at least $48 \mathrm{~h}$ after TC, using the dot-probe task, which is commonly used to assess biased processing (41). However, in the present design, we included before the dot-probe task, an extinction training and memory reinstatement. These manipulations after memory consolidation, may be responsible of the lack of an attentional bias as reported in Fernández and colleagues (36). In this sense, other authors showed that the number of extinction and reinstatement trials should be similar in order to recover the attentional bias. This premise was not fulfilled in the present experimental design, as we used 12 extinction trials and only 3 reinstatement trials.

Few reports used cognitive-interventions to engage and overload working-memory in reconsolidation studies. First, James and co-workers used an episodic memory based on "traumatic film". After memory-reactivation, subjects played a computer game (Tetris). They found a diminution in the number of memory intrusions of the "trauma film", compared with the control group. The target memory and the interfering task, required visuo-spatial working memory, thus the competition for similar resources may explain the weakened of the visual aspects of the intrusive memory. Using a similar target memory, Gotthard and Gura (42) used a video to create a positive 
declarative memory. After its reactivation, participants performed a visuospatial interference task (word search). This interference was effective in reducing free recall of the video details at testing. Finally, Chalkia and coworkers (28), found that an emotional working memory task with visuo-spatial processing, was unable to impair the re-stabilization of a threat memory. They proposed, that the negative results may depend on an inappropriate or low demanding working memory task. Here, regardless of the different nature of the threat-memory and HWM, we found a strong impairment on memory re-stabilization. The whole understanding of these finding, may arise from taking into consideration the different large-scale functional networks. The salience/defensive network shows similarities with the defensive survival circuits (3). Moreover, another large-scale network, is the central-executive control network, associated with cognitively demanding tasks such as working-memory maintenance and the reduction of subjective feelings of fear and anxiety $(32,43)$. Different reports, suggested that the salience/defensive survival-network and the central executive control network act as opposing systems (44). When participants perform a workingmemory task during a threatening situation, activation in the central-executive control network is reduced compared to a nonthreatening context. In line with these results, other report showed that the connectivity within the salience/defensive survival network increase during acute threat and decrease in the central-executive control network. Behaviorally, it was demonstrated that during TC acquisition is impaired, when simultaneously subjects perform a demanding working memory task (45).

This context supports the results obtained in this report. A HMW as the PASAT, may compete for neural resources impairing the threat-memory reconsolidation. We 
demonstrated that, the cognitive effort but not arousal levels during the HWM (Figure 3), may be related to the postulated effect of the salience/defensive survival-network and the central executive control-network on memory re-stabilization. Finally, the characteristics of our experimental design with healthy participants are in line with two main translational proposals in the field. First the use working-memory tasks to interfere emotional memories re-stabilization. Different interventions have been candidates such as the Eye Movement Desensitization and Reprocessing, Tetris game and the cognitive reappraisal technique, showed positive effects on emotional memory weakening $(27,46)$. However, the ideal candidate should consent to increase or decrease the cognitive demand. In this report, we showed that the cognitive effort of the working-memory task, could be monitored by online measures such as sympathetic activity. In addition, this effortful aspect of the working-memory task could be adjusted according to the protocol requirements such as the time interval between stimuli. To conclude, considering the unique complexities of anxiety disorders, it is necessary to add to animal models of anxiety disorders and clinical research with patients, the valuable resource of studies in healthy subjects. It offers a new inside named Experimental Psychopathology approach (47). This approach relies on a continuum from normal to pathological anxiety and considers the underlying mechanisms. Thus, the results obtained in this study disentangle the relation between the implicit memory, cognitive, valenced systems and the possibility to weaken both the threatening memory and the systems associated with the maintenance of anxiety profile. Future research may reveal if these effects also may be obtained in subjects with different anxiety profiles. 


\section{ACKNOWLEDGEMENTS AND DISCLOSURES.}

We thank Angel Vidal for technical assistance. This work was supported by Agencia Nacional de Promoción Científica y Tecnológica (http://www.agencia.mincyt. gob.ar/) PICT 2013-0412 and PICT 2016-0243 (MEP). The funders had no role in study design, data collection and analysis, decision to publish, or preparation of the manuscript. The authors declare no conflict of interest.

\section{REFERENCES.}

1. Kessler RC, Berglund P, Demler O, Jin R, Merikangas KR, Walters EE (2005):

Lifetime prevalence and age-of-onset distributions of DSM-IV disorders in the National Comorbidity Survey Replication. Arch Gen Psychiatry 62: 593-602.

2. Olatunji BO, Cisler JM, Tolin DF (2007): Quality of life in the anxiety disorders: a meta-analytic review. Clin Psychol Rev 27: 572-581.

3. LeDoux JE, Pine DS (2016): Using neuroscience to help understand fear and anxiety: a two-system framework. American journal of psychiatry 173: 1083-1093.

4. Rescorla RA (1988): Behavioral studies of Pavlovian conditioning. Annual review of neuroscience 11: 329-352.

5. Mineka S, Oehlberg K (2008): The relevance of recent developments in classical conditioning to understanding the etiology and maintenance of anxiety disorders. Acta Psychologica 127: 567-580.

6. Bouton ME, Mineka S, Barlow DH (2001): A modern learning theory perspective on the etiology of panic disorder. Psychological Review 108: 4-32. 
7. Grupe DW, Nitschke JB (2013): Uncertainty and anticipation in anxiety: an integrated neurobiological and psychological perspective. Nature Reviews Neuroscience 14: 488-501.

8. MacLeod C, Mathews A (2012): Cognitive bias modification approaches to anxiety. Annual Review of Clinical Psychology 8: 189-217.

9. Cisler JM, Koster EH (2010): Mechanisms of attentional biases towards threat in anxiety disorders: An integrative review. Clinical psychology review 30: 203216.

10. Coles ME, Heimberg RG (2002): Memory biases in the anxiety disorders: Current status. Clinical Psychology Review 22: 587-627.

11. Paulus MP, Stein MB (2006): An insular view of anxiety. Biological psychiatry 60: $383-387$.

12. Foa EB, Franklin ME, Perry KJ, Herbert JD (1996): Cognitive biases in generalized social phobia. Journal of abnormal psychology 105: 433.

13. McGaugh JL (2000): Memory--a century of consolidation. Science 287: 248-251.

14. Dudai Y (2012): The restless engram: consolidations never end. Annual review of neuroscience 35: 227-247.

15. Fernández RS, Boccia MM, Pedreira ME (2016): The fate of memory: reconsolidation and the case of prediction error. Neuroscience \& Biobehavioral Reviews 68: 423-441.

16. Nader K, Schafe GE, Le Doux JE (2000): Fear memories require protein synthesis in the amygdala for reconsolidation after retrieval. Nature 406: 722-726.

17. Lee JL (2009): Reconsolidation: maintaining memory relevance. Trends in neurosciences 32: 413-420. 
18. Nader K, Hardt O, Lanius R (2013): Memory as a new therapeutic target. Dialogues Clin Neurosci 15: 475-486.

19. Fernández RS, Pedreira ME, Boccia MM (2017): Does reconsolidation occur in natural settings? Memory reconsolidation and anxiety disorders. Clinical Psychology Review 57: 45-58.

20. Das RK, Lawn W, Kamboj SK (2015): Rewriting the valuation and salience of alcohol-related stimuli via memory reconsolidation. Translational psychiatry 5: e645.

21. Saladin ME, Gray KM, McRae-Clark AL, LaRowe SD, Yeatts SD, Baker NL, et al. (2013): A double blind, placebo-controlled study of the effects of post-retrieval propranolol on reconsolidation of memory for craving and cue reactivity in cocaine dependent humans. Psychopharmacology 226: 721-737.

22. Fernández RS, Bavassi L, Kaczer L, Forcato C, Pedreira ME (2016): Interference Conditions of the Reconsolidation Process in Humans: The Role of Valence and Different Memory Systems. Front Hum Neurosci 10. https://doi.org/10.3389/fnhum.2016.00641

23. Kindt M, Soeter M, Vervliet B (2009): Beyond extinction: erasing human fear responses and preventing the return of fear. Nature neuroscience 12: 256-258.

24. Brunet A, Saumier D, Liu A, Streiner DL, Tremblay J, Pitman RK (2018):

Reduction of PTSD Symptoms With Pre-Reactivation Propranolol Therapy: A Randomized Controlled Trial. AJP 175: 427-433.

25. Soeter M, Kindt M (2015): An Abrupt Transformation of Phobic Behavior After a Post-Retrieval Amnesic Agent. Biological psychiatry. Retrieved December 17, 2015 , from http://www.sciencedirect.com/science/article/pii/S0006322315003133 
26. Kindt M, Soeter M (2011): Reconsolidation in a human fear conditioning study: a test of extinction as updating mechanism. Biological psychology. Retrieved February 6, 2014, from http://www.sciencedirect.com/science/article/pii/S030105111100250X

27. James EL, Bonsall MB, Hoppitt L, Tunbridge EM, Geddes JR, Milton AL, Holmes EA (2015): Computer Game Play Reduces Intrusive Memories of Experimental Trauma via Reconsolidation-Update Mechanisms. Psychol Sci 26: 1201-1215.

28. Westbrook A, Braver TS (2015): Cognitive effort: A neuroeconomic approach. Cogn Affect Behav Neurosci 15: 395-415.

29. Chalkia A, Vanaken L, Fonteyne R, Beckers T (2019): Interfering with emotional processing resources upon associative threat memory reactivation does not affect memory retention. Scientific Reports 9: 1-11.

30. Morris JS, Friston KJ, Dolan RJ (1997): Neural responses to salient visual stimuli. Proceedings of the Royal Society of London Series B: Biological Sciences 264: 769-775.

31. Whalen PJ, Rauch SL, Etcoff NL, McInerney SC, Lee MB, Jenike MA (1998): Masked Presentations of Emotional Facial Expressions Modulate Amygdala Activity without Explicit Knowledge. J Neurosci 18: 411-418.

32. Daniel TA, Katz JS, Robinson JL (2016): Delayed match-to-sample in working memory: A BrainMap meta-analysis. Biological Psychology 120: 10-20.

33. de Voogd LD, Hermans EJ, Phelps EA (2018): Regulating defensive survival circuits through cognitive demand via large-scale network reorganization. Current Opinion in Behavioral Sciences 24: 124-129.

34. Craske MG, Stein MB (2016): Anxiety. The Lancet 388: 3048-3059. 
35. Insel T, Cuthbert B, Garvey M, Heinssen R, Pine DS, Quinn K, et al. (2010):

Research Domain Criteria (RDoC): Toward a New Classification Framework for Research on Mental Disorders. Am Psychiatric Assoc. Retrieved August 8, 2017, from

http://ajp.psychiatryonline.org/doi/full/10.1176/appi.ajp.2010.09091379

36. Fernández RS, Picco S, Messore F, Pedreira ME (2018): Effects of Threat

Conditioning on the Negative Valanced Systems and Cognitive Systems.

Scientific Reports 8: 1-9.

37. Grupe DW (2017): Decision-Making in Anxiety and Its Disorders.

38. Moutoussis M, Story GW, Dolan RJ (2015): The computational psychiatry of reward: broken brains or misguided minds? Front Psychol 6.

https://doi.org/10.3389/fpsyg.2015.01445

39. Gronwall DM (1977): Paced auditory serial-addition task: a measure of recovery from concussion. Percept Mot Skills 44: 367-373.

40. Mineka S, Zinbarg R (2006): A contemporary learning theory perspective on the etiology of anxiety disorders: it's not what you thought it was. Am Psychol 61: $10-26$.

41. Posner MI (1980): Orienting of Attention. Quarterly Journal of Experimental Psychology 32: 3-25.

42. Gotthard GH, Gura H (2018): Visuospatial word search task only effective at disrupting declarative memory when prediction error is present during retrieval. Neurobiology of Learning and Memory 156: 80-85.

43. Etkin A, Büchel C, Gross JJ (2015): The neural bases of emotion regulation. Nat Rev Neurosci 16: 693-700. 
44. Hermans EJ, Henckens MJAG, Joëls M, Fernández G (2014): Dynamic adaptation of large-scale brain networks in response to acute stressors. Trends Neurosci 37: 304-314.

45. Carter RM, Hofstötter C, Tsuchiya N, Koch C (2003): Working memory and fear conditioning. Proc Natl Acad Sci U S A 100: 1399-1404.

46. de Voogd LD, Kanen JW, Neville DA, Roelofs K, Fernández G, Hermans EJ (2018): Eye-Movement Intervention Enhances Extinction via Amygdala Deactivation. J Neurosci 38: 8694-8706.

47. Grillon C, Robinson OJ, Cornwell B, Ernst M (2019): Modeling anxiety in healthy humans: a key intermediate bridge between basic and clinical sciences. Neuropsychopharmacology 44: 1999-2010. 


\section{FIGURE LEGENDS.}

Figure 1. Schematic representation of the experiment. (A) Group description across the 3 Days. HMW stands for "Highly Demanding Working Memory Task" and LMW for "Low Demanding Working Memory Task". (B) Experimental timeline for the 3 groups.

Figure 2. Reconsolidation interference of Threat Memory. (A) Reactivation-HWM group. (B) noReactivation-HWM. (C) Reactivation-LWM. Mean SCR $(\mu \mathrm{S}) \pm \mathrm{SEM}$. Left panel: Threat conditioning acquisition on Day 1. Middle panel: Reactivation (RC) on Day 2. Right panel: Extinction training and reinstatement (RST) on Day 3. ${ }^{*} \mathrm{P}<0.05$.

Figure 3. Subjects arousal and performance during the Working Memory Intervention (Day 2). (A) Mean SCL $(\mu S) \pm S E M$ across time for each group during the HWM or LWM. (B) Proportion of correct responses ( \pm SEM) for each group during the HWM or LWM. (C) Correlation Matrix between the working memory interventions on Day 2 (SCL and Proportion of correct responses) and TC memory on Day 3 (Mean SCR difference and Reinstatement) (D) Arousal vs Performance correlation. (E) TC retention vs Performance correlation. ${ }^{*} \mathrm{P}<0.05$.

Figure 4. Cognitive and negative-valenced systems evaluation after threat conditioning testing (Day 3). (A) Stimuli Representation (aversiveness). Mean score ( \pm SEM) difference between CS+ and CS-. (B) Stimuli Generalization. Mean score ( \pm SEM) 
for non-trained negative and neutral pictures. (C) Valuation: Probability (left panel) and Cost (right panel). Mean score ( \pm SEM) difference between positive and negative scenarios for CS+ and CS-. (E) Attentional bias (dot-probe). Difference in ms. between incongruent and congruent trials for 3 types of stimuli compounds (CS+ vs CS-, CS+ vs CSn and CS- vs CSn). $*$ P $<0.05$ 

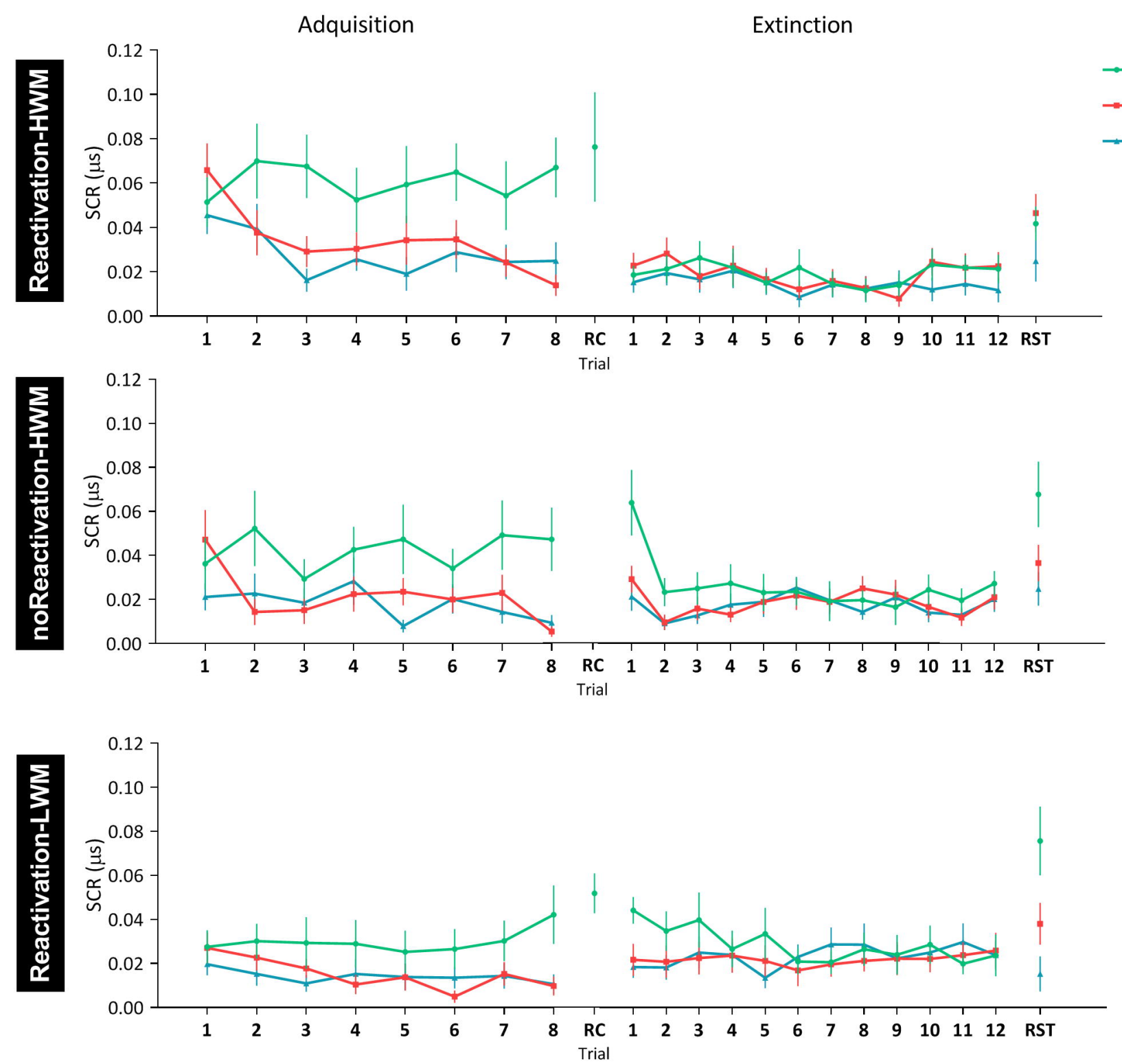


\section{A}

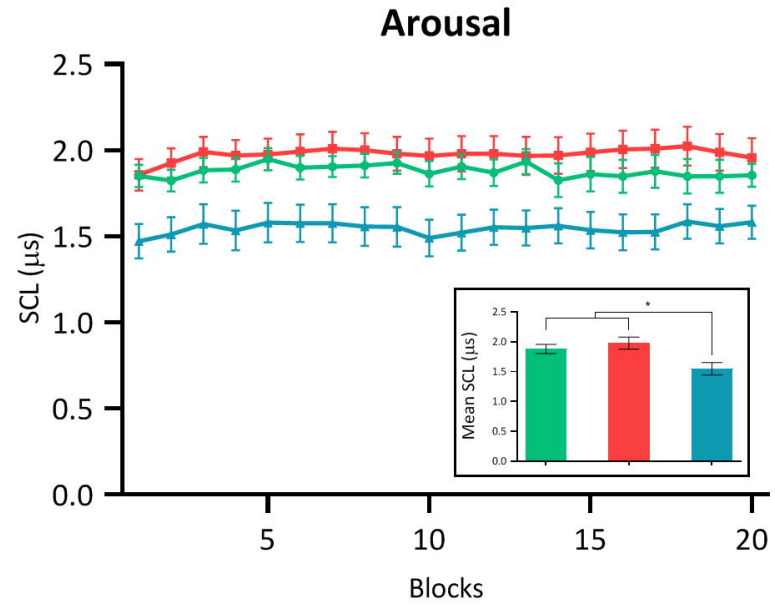

B

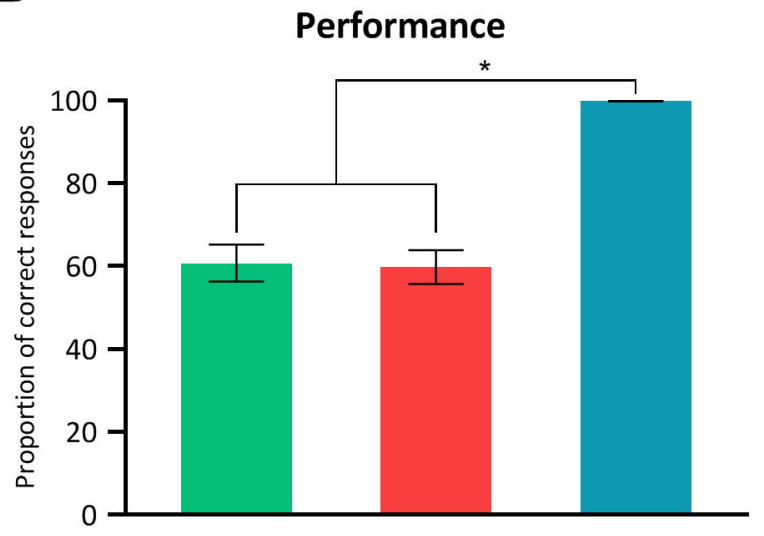

C

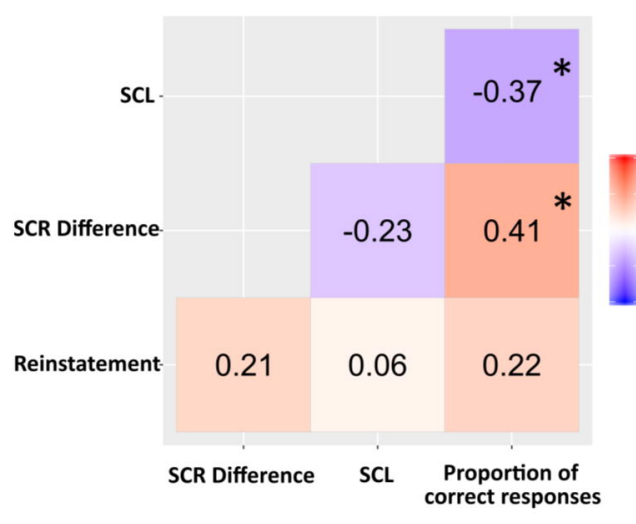

D

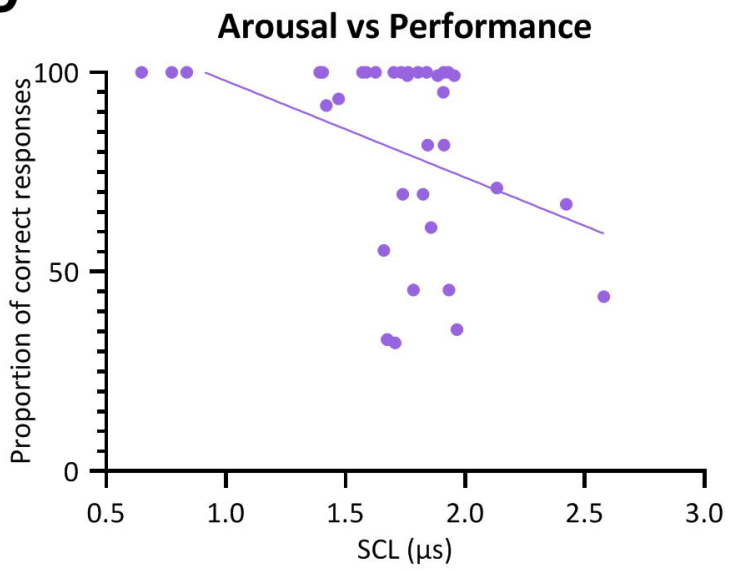

E

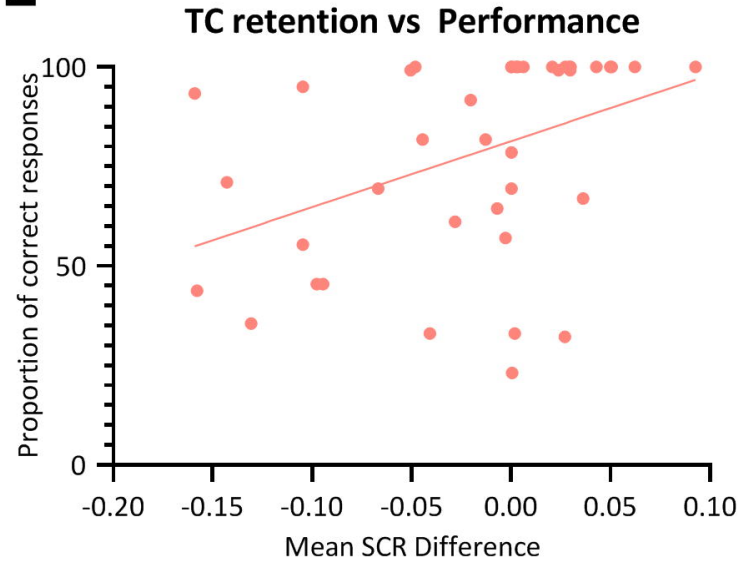


A

Stimuli Representation

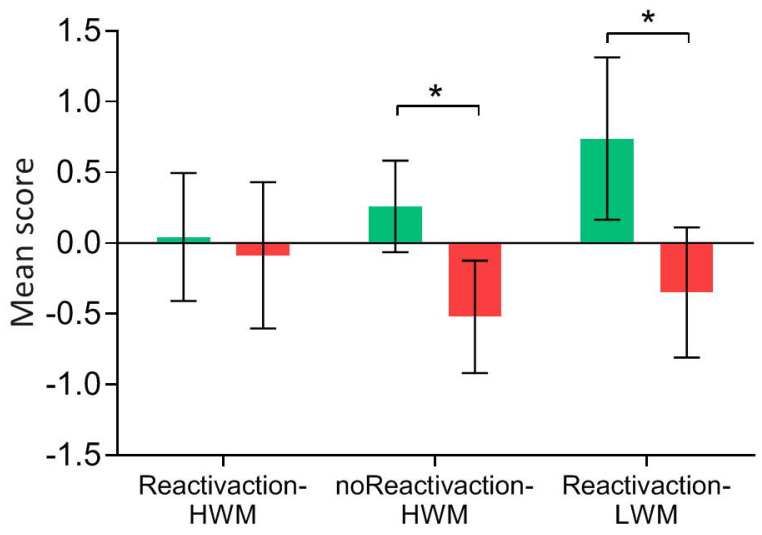

C

Probability

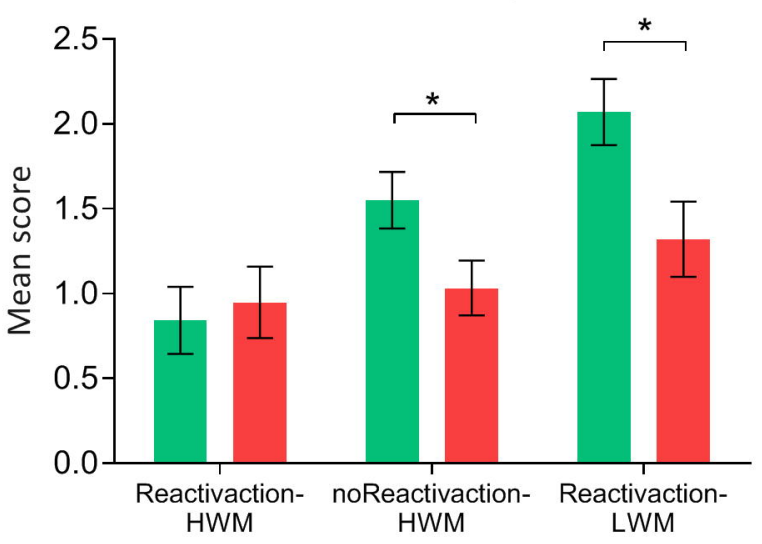

\section{B}

Stimuli Generalization

CS+

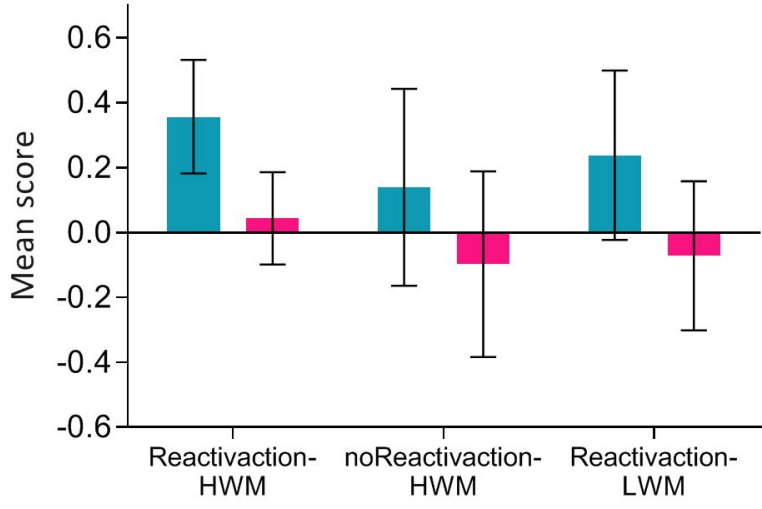

Aversive

- Neutral
D

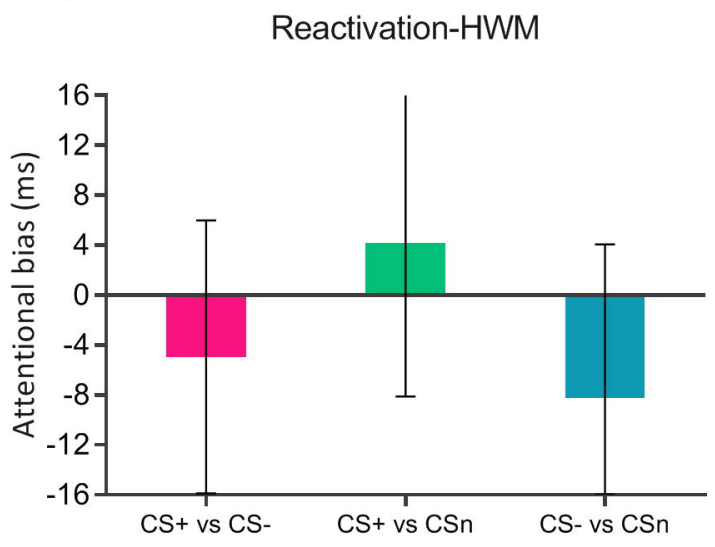

\section{Attentional bias}

noReactivation-HWM

Reactivation-LWM
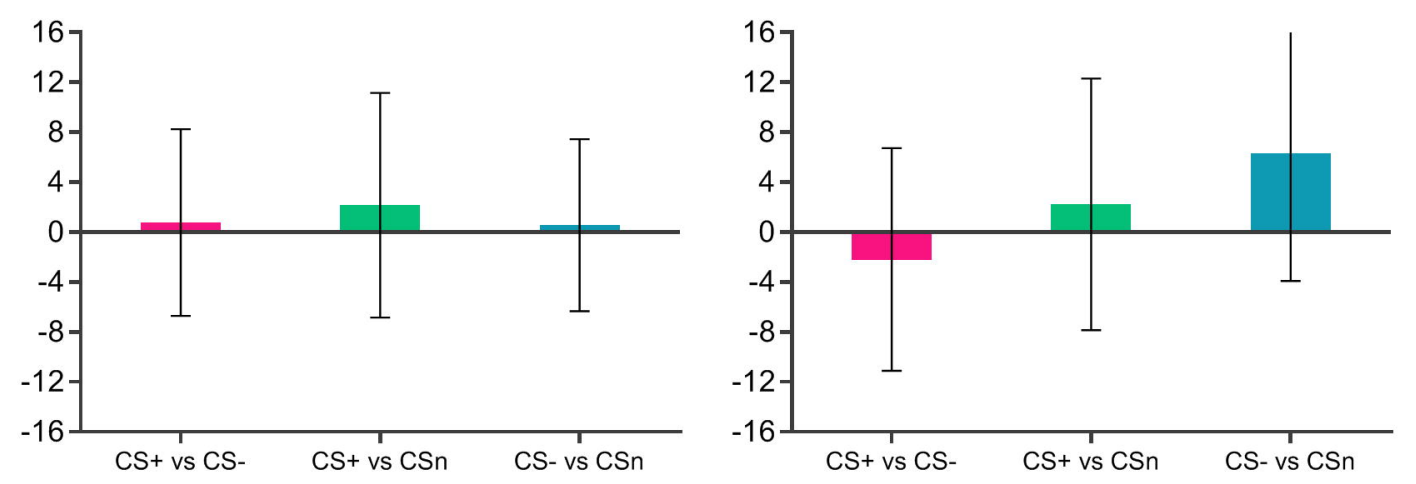